\title{
Wirtschaftlichkeitsverfahren, die keine sind: rechtliches Gutachten im Licht unabhängiger Statistik
}

\section{Michel Romanens}

Vom Autor et al. bisher zu diesem Thema in der SÄZ erschienen:

Krankenkassen, santésuisse und Wirtschaftlichkeitsverfahren, die keine sind ... Schweiz Ärztezeitung. 2009;90(1/2): 49-52.

Vergleichbarkeit der von Schweizer Ärztinnen und Ärzten verursachten Behandlungskosten in der Grundversorgung. Schweiz Ärztezeitung. 2010;91(35):1342-6.

\section{Die Literaturangaben finden sich im Internet unter www.saez.ch $\rightarrow$ Aktuelle Nummer oder $\rightarrow$ Archiv $\rightarrow$ $2011 \rightarrow 9$.}

Korrespondenz:

Dr. med. Michel Romanens Spezialarzt FMH Innere Medizin und Kardiologie

Ziegelfeldstrasse 1

CH-4600 Olten

info@kardiolab.ch

www.vems.ch

\section{Einführung}

Am 1. September 2010 publizierte der Verein Ethik und Medizin Schweiz (VEMS) zwei von ihm in Auftrag gegebene statistische Gutachten, die insgesamt das Vorgehen der santésuisse im Bereich der Wirtschaftlichkeitsverfahren als «nicht sachgerecht» beurteilten. Insbesondere wurde dabei auch das Problem der impliziten Rationierung erstmals in aller Deutlichkeit thematisiert: Das Verfahren der santésuisse erzeugt Angst, erfasst die Falschen und schafft faktisch ein irrationales Globalbudget in der medizinischen Grundversorgung mit der impliziten Gefahr versteckter Rationierung und bedrohter Patientensicherheit.

Wie weit die Rechtsgrundlagen für einen solchen massiven Eingriff in die Behandlungsnotwendigkeit unserer Patientinnen und Patienten überhaupt existieren, ist zentraler Gegenstand des Gutachtens von PD Dr. iur. Ueli Kieser, Vizedirektor am Institut für Rechtswissenschaften und Rechtspraxis an der Universität St. Gallen, das dem Verein Ethik und Medizin seit Ende Januar 2011 vorliegt. Es beleuchtet die Rechtslage im Licht der erwähnten beiden statistischen Gutachten. Im Folgenden fasse ich die Resultate zusammen und wage einen Blick in die nahe Zukunft, denn schon im April 2011 wird der Nationalrat über Gesetzeserweiterung zu entscheiden haben, wonach im neuen Absatz 6 des Artikels 56 KVG festgeschrieben werden soll, dass künftig die Wirtschaftlichkeitsverfahren zusammen mit Vertretern der Ärzteschaft durchzuführen seien. Es geht jetzt also um die «Wurst».

\section{Grundlegende Rechtsaspekte}

Das Gutachten «Wirtschaftlichkeitsverfahren in der Krankenversicherung, neueste Entwicklungen und eine Würdigung» wurde vom Verein Ethik und Medizin am 8. November 2010 in Auftrag gegeben. Gemäss Auftrag soll eine Analyse der aktuellen Rechtsprechung unter Berücksichtigung der beiden Gutachten von Prof. Wasem [1]* und PD Schwenkglenks [2].

Unbestritten bleibt die hohe Bedeutung der Wirtschaftlichkeit in der Sozialversicherung [3]. Das Schweizerische Krankenversicherungssystem will eine qualitativ hochstehende gesundheitliche Versorgung zu möglichst günstigen Kosten sicherstellen [4].

\section{Des procédures d'économicité qui} n'en sont pas: une expertise juri-

\section{dique à la lumière de statistiques indépendantes}

L'expertise mandatée par l'association VEMS (éthique et médecine Suisse) pointe du doigt, aussi bien sur le plan juridique que statistique, les procédures d'économicité telles que les pratique santésuisse.

Les expertises de Schwenkglenks [2], Wasem [1] et Kieser [30] montrent que la méthode utilisée pour calculer la moyenne des coûts comporte d'importantes lacunes sur le plan juridique et statistique. Cette méthode ne permet pas de prouver des pratiques non économiques de la part des médecins dans le traitement des patients. L'index ANOVA est tout aussi remis en question. Quant à savoir si ces procédures de vérification disposent d'une base juridique suffisante, le doute subsiste.

Par ailleurs, il est exclus - s'il n'est pas possible d'avoir une vue d'ensemble suffisante des effets compensatoires - de pouvoir procéder à une évaluation globalement convaincante de l'économicité de I'activité médicale. De plus, les particularités d'un cabinet médical devraient pouvoir être clarifiées d'office. Il est en effet erroné de parler de preuve principale et de contre-preuve. Par ailleurs, un nouvel arrêt du Tribunal fédéral de décembre 2010 [31] stipule que c'est à santésuisse, et avec elle, aux assureurs-maladie, de fournir au médecin attaqué (pour avoir trop prescrit) les données des médecins formant le groupe de comparaison. L'égalité des armes s'en trouve ainsi améliorée, mais le fardeau de la preuve est encore à la charge du médecin mis en cause. 
Zudem wirkt sich das Wirtschaftlichkeitsgebot insofern auf den Leistungsbereich der Krankenversicherung aus, als das Kosten-/Nutzenverhältnis bzw. das Kosten-/Wirksamkeitsverhältnis im Einzelfall berücksichtigt wird [5].

Das Gebot zu wirtschaftlichem Handeln gemäss Art. $56 \mathrm{KVG}$ richtet sich an alle Leistungserbringer [6]. Eine Leistung, die im Rahmen der Krankversicherung erbracht wird, hat wirksam, zweckmässig und wirtschaftlich zu sein (Art. 32 KVG) [7]. Die Vergütung von unwirtschaftlichen Leistungen kann gemäss Gesetz zurückgefordert werden. Wie diesbezüglich jedoch konkret vorzugehen ist, hat der Gesetzgeber nicht genauer geregelt [8]

\section{Durchschnittkostenvergleiche ohne Berücksichtigung kompensatorisch eingesparter Kosten erlauben keine haltbare Aussage zur Wirtschaftlichkeit von Leistungen.}

Obwohl die Einzelfallkontrolle das primäre gesetzliche System darstellt, sind die Krankenversicherer zu Pauschalprüfungen basierend auf Vergleichszahlen übergegangen, wobei durch die Rechtsprechung praktisch ausschliesslich der arithmetische Mittelwertvergleich berücksichtigt wird [8]. Das Bundesgericht betrachtet diesen Vergleich als wissenschaftlich korrekt und lehnt konstant den Einwand ab, dass die Wirtschaftlichkeit der Behandlungsweise nicht nach der statistischen, sondern nach der analytischen Methode beurteilt werden müsse [9]. Es vertritt diesbezüglich den Standpunkt, dass die Zulässigkeit der statistischen Methode unter Zugrundelegung der Statistik santésuisse in der Rechtsprechung anerkannt sei und die kantonalen Gerichte in der Wahl der Prüfungsmethode frei seien [10]. Die statistische Methode ist nur aussagekräftig, wenn die wesentlichen Praxismerkmale der Vergleichsgruppe untereinander und mit der Praxis des geprüften Arztes übereinstimmen $[11,13]$. Zudem müssen die direkten und veranlassten Kosten berücksichtigt werden [12, 14]. Eine Entscheidung aufgrund geheim gehaltener Akten ist ausgeschlossen $[15,16]$. Eine eigentliche Beweislast gibt es im Sozialversicherungsrecht, im Gegensatz zum Zivilrecht, nicht. Die massgebenden Sachverhaltselemente müssen von Amtes wegen ermittelt werden; die Beweise werden frei gewürdigt [17].

\section{Problematische Aspekte}

Die Entwicklung in den letzten Jahren zeigt, dass die Wirtschaftlichkeitsprüfung beim Leistungserbringer an Bedeutung verliert. Gründe dafür sind: a) Immer mehr Leistungen werden durch die Rechtssetzung einer besonderen vertrauensärztlichen Prüfung und einer darauf gestützten vorgängigen Kostengutspra- che unterstellt; b) Bessere Leistungserfassungssysteme der Krankenversicherer ermöglichen bessere und umgehende Kontrolle der Einzelleistung; c) Zunahme der Patientenfluktuation; Patienten suchen häufig verschiedene Ärzte auf; d) Aufgrund des Rechtsgleichheitsgebotes müssten eigentlich alle Leistungserbringer, nicht nur die ambulant tätigen erfasst werden; e) Zunehmende Subspezialisierung bei gleichem Facharzttitel erschwert die Vergleichbarkeit immer mehr.

Der Durchschnittskostenvergleich ist dann eine verlässliche Methode für die Beurteilung der Wirtschaftlichkeit, wenn die zugrunde gelegten Daten verlässlich und vollständig sind. Die Datenqualität wird jedoch zunehmend in Frage gestellt. Gründe dafür sind zu Beispiel: a) Die Datenqualität betreffend Verlässlichkeit und Validierung wird zunehmend in Frage gestellt [2]; b) Die Leistungen sind eigentliche Pflichtleistungen, der Beweis, dass dem nicht so ist, wird unter Umständen durch die Krankenversicherer nicht rechtsgenügend dargelegt; c) Zudem unterliegt die ärztliche Tätigkeit grundsätzlich der Wirtschaftsfreiheit [18]; d) Zudem meint der Gesetzgeber die Erfassung der Wirtschaftlichkeit bei der Behandlung einer kranken Person, womit der Einzelarzt im Rahmen der Wahlfreiheit des Patienten betreffend Wirtschaftlichkeitsverfahren allenfalls verzerrt erfasst wird [1, 2]; e) ungenügend berücksichtigt werden Tiers-payant-Situationen und Franchisenanteile [19]; f) Die zu berücksichtigenden Gesamtkosten werden ungenügend erfasst, insbesondere betreffend veranlasste und eingesparte Kosten. Durchschnittkostenvergleiche ohne die Berücksichtigung der kompensatorisch eingesparten Kosten erlauben grundsätzlich keine haltbare Aussage zur Wirtschaftlichkeit von Leistungen; g) Die Bildung von Vergleichsgruppen aufgrund des FMH-Titels kann ungenügend sein, da bestimmte fachärztliche Ausrichtungen zu erheblichen Unterschieden in der Tätigkeit und damit in der Art der behandelten Krankheiten führt [20, 21] oder da ein Facharzt mit Subspezialität auch Allgemeine Innere Medizin praktiziert [22]; h) Zudem müssen die Vergleichsgruppen genügend gross sein [23]; i) In kleineren Arztpraxen mit weniger Patientinnen und Patienten werden Hochkosten-Patienten schlechter abgepuffert [1].

Die ANOVA-Methode ist für die Beurteilung der Wirtschaftlichkeit ebenfalls nicht rechtsgenügsam: a) die Methode erzeugt für die Korrektur des Alters und Geschlechts fiktive Werte im Rahmen eines gesamtschweizerischen Indexes und verunmöglicht die Bezugnahme auf konkrete Tatsachen und eliminiert regionale Kostenunterschiede [24]; b) damit wird indirekt - da in der Risikoadjustierung nicht berücksichtigt - das Morbiditätsrisiko auf den Arzt abgewälzt [1]; c) Medikamentenabgabe und Selbstzahleranteil werden ungenügend berücksichtigt [2]; d) weitere Praxisbesonderheiten müssen vom behandelnden Arzt mittels «Gegenbeweis» geltend gemacht werden, da die Wirtschaftlichkeit nicht mit einem 
Vergleich der beiden Zahlen bestimmt werden kann, gleichzeitig ist es aber problematisch, wenn die Versicherer selbst nicht in der Lage sind, diesen Beweis zu erbringen.

Die gesetzliche Grundlage für die Rückforderungen bei unwirtschaftlichem Verhalten ist ungenügend. Solche Rückforderungen greifen in die Eigentumsgarantie und in Aspekte der Wirtschaftsfreiheit ein. Für einen solchen Eingriff bedarf es einer hinreichenden gesetzlichen Grundlage. Die Anforderungen an die gesetzliche Grundlage sind umso höher, je schwerer der Eingriff ist. Sie muss hinreichend bestimmt und klar sein. Art. 56 KVG erschöpft sich darauf, festzustellen, dass eine zu Unrecht bezahlte Vergütung zurückgefordert werden kann, und bestimmt, wer rückforderungsberechtigt ist. Diese Gesetzesnorm ist somit sehr knapp gehalten und zeigt lediglich den Grundsatz sowie die zur Rückforderung berechtigte Partei auf. Es fehlen Angaben bezüglich der Art und Weise, wie die Rückforderung zu bestimmen ist, welche Leistungen einbezogen werden können, wie die Unwirtschaftlichkeit zu bestimmen ist und wie das Verhältnis zu sonstigen prinzipiellen Aspekten (z.B. der Therapiewahlfreiheit) ist [26-29].

\section{Schlussfolgerungen}

Die Gutachten Schwenkglenks [2], Wasem [1] und Kieser [30] zeigen, dass die Durchschnittskostenmethode sowohl in rechtlicher wie in statistischer Sicht an wesentlichen Mängeln leidet. Die Methode ist nicht geeignet, den Beweis zu erbringen, dass ein

\section{Das Papier der Arbeitsgruppe WZW der FMH zeigt klar, welche Wege - unter Einbezug von Morbiditäts- faktoren - zu beschreiten sind.}

unwirtschaftliches Handeln bei der Behandlung von Patientinnen und Patienten vorliegt. Dies gilt auch für den ANOVA-Index. Es bestehen Zweifel, dass diese Prüfverfahren eine genügende Rechtsgrundlage aufweisen.

Zudem ist es - wenn nicht eine hinreichende Übersicht über die kompensatorischen Effekte möglich ist - ausgeschlossen, eine insgesamt überzeugende Beurteilung der Wirtschaftlichkeit der ärztli- chen Tätigkeit vorzunehmen. Praxisbesonderheiten müssten ferner von Amtes wegen abgeklärt werden. Es ist verfehlt, von Haupt- und Gegenbeweis zu sprechen. Zudem verlangt ein neuer Bundesgerichtsentscheid vom Dezember 2010 [31], dass die santésuisse und mit ihr die Krankenversicherer dem wegen Überarztung vor Gericht stehenden Arzt die Daten zur Vergleichsgruppe offenlegen müssen. Damit wird die Waffengleichheit verbessert, die Beweislast liegt aber weiter beim angeschuldigten Arzt.

\section{Ausblick}

Die unsachgemässe Durchführung der Wirtschaftlichkeitsverfahren durch die santésuisse ist aufgrund der vom Verein und Medizin Ethik Schweiz in Auftrag gegebenen drei Gutachten sowohl in statistischer wie rechtlicher Hinsicht erwiesen. Die von der Nationalratskommission Soziales und Gesundheit an den Nationalrat überwiesene parlamentarische Initiative Heim/Cassis/Meyer fordert folgerichtig und überparteilich [32] eine Gesetzesänderung im Art. 56 KVG: die Prüfungen hätten zusammen mit den Ärztinnen und Ärzten zu erfolgen. Erst diese Änderung würde es ermöglichen, die von niemandem bestrittene Notwendigkeit zur Durchführung von Wirtschaftlichkeitsverfahren korrekt und professionell durchzuführen. Das Papier der Arbeitsgruppe WZW der FMH zeigt klar, welche Wege - unter Einbezug von Morbiditätsfaktoren - zu beschreiten sind, insbesondere der Einbezug von Morbiditätsvariablen im Sinne eines sachgerechten Risikoausgleichs für notwendige Abklärungs- und Behandlungskosten [33]. Und last but not least: Mit Bundesgerichtsentscheid vom 19. Januar 2011 bleibt der Gesamtkostenindex auch in Zukunft für die Beurteilung der Wirtschaftlichkeit massgebend. Die veranlassten Kosten spielen für die Berechnung des Gesamtkostenindex weiterhin eine bestimmende Rolle. Neu ist, dass eine Rückforderung nur auf den direkten Kosten erhoben werden kann [34]. Damit wird das Verfahren weiterhin grundsätzlich nicht in Frage gestellt, das «Bussgeld» jedoch reduziert. Und: In einem weiteren Urteil kam das Bundesgericht zum Schluss, dass ein Durchschnittskostenvergleich unmöglich sei, wenn zwischen den Praxisbesonderheiten der Vergleichsgruppe und des kontrollierten Arztes ein zu grosser Unterschied bestehe. In einem solchen Fall müsse die analytische Methode angewandt werden [35]. 


\section{Literatur}

1 Prof. Dr. Jürgen Wasem, Lehrstuhl für Medizinmanagement, Universität Duisburg-Essen, in Kooperation mit ForBiG GmbH. Forschungsnahe Beratungsgesellschaft im Gesundheitswesen GmbH: Beurteilung des Screening-Verfahrens der Krankenversicherer in der Schweiz zur Identifikation von Überarztung; 26. Juli 2010, S. 1-38. http://physicianprofiling.ch/ gutachtenwasem2010.pdf

2 PD Dr. Matthias Schwenkglenks, MPH, Institute of Pharmaceutical Medicine / ECPM, University of Basel: Vergleich verschiedener Instrumente (Rechnungstellerstatistik der santésuisse und Praxisspiegel der Trustcenter) zur Beurteilung der von Schweizer Ärzten in der Grundversorgung verursachten Behandlungskosten; Statistischer Studienbericht; 8. Juli 2010. S. 1-75. http://physicianprofiling.ch/ rsspssstatistikbericht052010.pdf

3 Vgl. dazu etwa Haller, Kölz, Gächter. Allgemeines Staatsrecht, wo sich der Begriff der Wirtschaftlichkeit nicht findet; analog Häfelin, Müller, Uhlmann. Verwaltungsrecht

4 Eugster. Krankenversicherung. N 10, mit Hinweis auf BGE 123 V 290, 127 V 80 sowie 130 V 196.

5 Bundesgerichtsurteil vom 23. November 2010, 9C 334/2010.

6 Urteil 9C_397/2009, E. 4.2.

7 Urteil 9C_334/2010, E. 7.7.

8 Eugster. Überarztung aus juristischer Sicht. S. 103 ff.

9 Urteil 9C_393/2007, E. 4.4; vgl. zudem das Grundsatzurteil 9C_968/2009.

10 Urteile des Bundesgerichts K 46/04, E. 5.1, K 9/07, E. 8.3.

11 Eugster. Überarztung aus juristischer Sicht, 104; Prinzip der «Homogenität».

12 BGE 133 V 37.

13 Eugster. Rechtsprechung des Bundesgerichts zum KVG. S. $391 \mathrm{f}$

14 RKUV 1999 320, E. 5.d und 5.e. / Vgl. dazu Art. 2 ff KLV.

15 BGE 132 V 387. / Zu berücksichtigen ist, dass es sich in Art. 47 ATSG um die verfahrensrechtliche Akteneinsicht und nicht um die datenschutzrechtliche Akteneinsicht handelt.

16 Vgl. dazu Art. 48 ATSG; die praktische Bedeutung dieser besonderen Akteneinsicht beschränkt sich auf eng abgegrenzte Bereiche (beispielsweise in bestimmten Fällen der Militärversicherung oder bei Fabrikationsgeheimnissen).

17 Randtitel des 6. Abschnittes vor Art. 56 KVG.

18 Vgl. etwa RKUV 1999 169; vgl. auch Eugster. Krankenversicherung, N 309, der darauf hinweist, dass die Tätigkeit des Arztes grundsätzlich der Wirtschaftsfreiheit untersteht.

19 Eugster. Überarztung aus juristischer Sicht, S. 111.

20 Dies stellt eine in verschiedener Hinsicht bedeutsame Ausgangslage dar. Vgl. zu den Versuchen, bestimmte Faktoren mit dem ANOVA-Index zu korrigieren Ziff. 6.3.3; hier wird aber auch darauf hinzuweisen sein, dass wegen der Nichtberücksichtigung der Morbidität der ANOVA-Index keine hinreichende Korrekturmöglichkeit darstellt.
21 Dazu Eugster. Überarztung aus juristischer Sicht. S. 105, mit Hinweis auf Nadig. Rationierung. S. 855.

22 So die Feststellung bei Eugster. Überarztung aus juristischer Sicht. S. 104 unten.

23 Dazu Eugster Überarztung aus juristischer Sicht. S. 107.

24 Eugster. Überarztung aus juristischer Sicht. S. 124 ff.

25 Verneinend - mit Hinweisen auf Gattiker und Iselin Eugster, Überarztung aus juristischer Sicht. S. $132 \mathrm{f}$.

26 Eugster. Überarztung aus juristischer Sicht. S. 135.

27 Rainer, Schweizer. St. Galler Kommentar zur BV, Art. $36 \mathrm{~N} 12$.

28 Eugster. Überarztung aus juristischer Sicht. S. 135 f., mit Hinweisen auf Gattiker und Iselin. Der genannte Autor bezieht sich aber nur auf die veranlassten Leistungen, ohne dass er näher auf die Frage einginge, ob nicht die gesamte Ausgestaltung der Rückforderung im heutigen Zeitpunkt eine ungenügende gesetzliche Grundlage hat.

29 Müller. Vermutung. 553.

30 www.physicianprofiling.ch/kieser2011.pdf

31 BGE 20101215 9C_968-2009, siehe dazu auch den Artikel von V. Junod, Seite 336 dieser Ausgabe.

32 Mitunterzeichnerinnen und -unterzeichner der Parlamentarischen Initiative Cassis/Heim/Meyer: Abate Fabio, Amherd Viola, Aubert Josiane, Banga Boris, Barthassat Luc, Beck Serge, Béguelin Marlyse, Berberat Didier, Bortoluzzi Toni, Bruderer Pascale, Brun Franz, Burkhalter Didier, Carobbio Guscetti Marina, Cassis Ignazio, Cathomas Sep, Daguet André, Donzé Walter, Dunant Jean Henri, Edith Günter Paul, Eggly Jacques-Simon, Fehr Jacqueline, Fluri Kurt, Frösch Therese, Galladé Chantal, Garbani Valérie, Germanier Jean-René, Glanzmann-Hunkeler Ida, Graf-Litscher Edith, Guisan Yves, Gutzwiller Felix, Gysin Remo, Häberli-Koller Brigitte, Hany Urs, Heim Bea, Hofmann Urs, Huber Gabi, Hubmann Vreni, Ineichen Otto, Jermann Walter, John-Calame Francine, Kleiner Marianne, Kohler Pierre, Lang Josef, Lustenberger Ruedi, Markwalder Christa, Marty Kälin Barbara, Maury Pasquier Liliane, Meyer-Kaelin Thérèse, Michel Jürg, Moret Isabelle, Müller Philipp, Müller Thomas, Müller-Hemmi Vreni Nordmann Roger, Pedrina Fabio, Rechsteiner Paul, Rechsteiner Rudolf, Rennwald Jean-Claude, Rey Jean-Noël, Robbiani Meinrado, Rossini Stéphane, Roth-Bernasconi Maria, Ruey Claude, Savary Géraldine, Schelbert Louis, Schenker Silvia, Simoneschi-Cortesi Chiara, Stahl Jürg, Steiert Jean-François, Stöckli Hans, Stump Doris, Teuscher Franziska, Thanei Anita, Triponez Pierre, Vaudroz René, Vermot-Mangold Ruth-Gaby, Vischer Daniel, Vollmer Peter, Widmer Hans, Zemp Markus.

33 www.fmh.ch/files/pdf4/FMH_Tarifdienst_WZW_ Positionspapier_D.pdf\&pli=1

34 BGE 9C_733/2010 vom 19.01.2011, Erw. 2.5.5.

35 BGE 9C 167/2010 vom 14.01.2011, Erw. 4.3. 